Revue d'histoire de l'Amérique française

REVUE D.HISTOIRE DE L'AMÉRIQUE FRANÇAISE

\title{
Le Père Éphrem Longpré tel que je l'ai connu
}

\section{Stéphane-Joseph Piat}

Volume 20, numéro 3, décembre 1966

URI : https://id.erudit.org/iderudit/302585ar

DOI : https://doi.org/10.7202/302585ar

Aller au sommaire du numéro

Éditeur(s)

Institut d'histoire de l'Amérique française

ISSN

0035-2357 (imprimé)

1492-1383 (numérique)

Découvrir la revue

Citer cet article

Piat, S.-J. (1966). Le Père Éphrem Longpré tel que je l'ai connu. Revue d'histoire de l'Amérique française, 20(3), 349-365. https://doi.org/10.7202/302585ar d'utilisation que vous pouvez consulter en ligne.

https://apropos.erudit.org/fr/usagers/politique-dutilisation/ 


\section{LE PERE EPHREM LONGPRÉ TEL QUE JE L'AI CONNU}

Quand se répandit la nouvelle du décès du Père Ephrem Longpré, les frères mineurs au courant de ses travaux et de ses projets déclarèrent unanimement: "C'est une perte irréparable pour l'Ordre. Cet homme-là ne sera pas remplacé." Un jeune religieux qui appartient à l'équipe des chercheurs du Collège International Saint-Bonaventure de Quaracchi m'écrivait au sujet du défunt: "C'est à lui que les Franciscains doivent en bonne partie la redécouverte du patrimoine de notre spiritualité bonaventurienne. Son œuvre restera un beau témoignage du franciscanisme de notre époque."

Une amitié longue et fidèle me vaut d'être invité à consacrer quelques pages à celui à qui je vouais une véritable admiration. Il ne s'agira pas, à proprement parler, d'un article biographique méthodiquement construit, mais plutôt d'un portrait étayé par les souvenirs personnels et les confidences reçues. Dans ses dernières années notamment, le Père Ephrem aimait revenir sur le passé. Il apportait à le faire un mélange de finesse, de fraîcheur et de candeur qui, malgré l'extrême vélocité du débit, donnait au récit un charme inimitable.

$\mathrm{Au}$ point de départ, une de ces familles patriarcales qui sont l'honneur du Canada. Issu d'une lignée de cultivateurs, établie outre-mer dès la fin du dix-septième siècle, Joseph Longpré s'était transplanté à Woonsocket, aux États-Unis, dans le Rhode Island. C'est là que son épouse, née Adéline Richard, mit au monde, le 24 août 1890, le premier de ses douze enfants, qui reçut au baptême le nom de Zéphirin. Quatre ans plus tard, le foyer regagnait la patrie et s'installait à Saint-Ephrem d'Upton, au Québec. 
Il fallait entendre notre franciscain évoquer la vie pauvre et laborieuse qu'on menait dans une habitation rurale, en lisière de la forêt, la frugalité des repas, l'austérité des mœurs, la rigueur du travail: le tout assaisonné de tendresse et de bonne humeur. L'image de la Sainte-Famille présidait aux devoirs d'état. Chaque jour, on récitait ensemble le chapelet, suivi des litanies des Saints. Le mois de Marie était célébré parmi les fleurs et les lumières. La maman était de ces femmes dont René Bazin a pu dire "qu'elles ont une âme sacerdotale et qu'elles la passent à leurs enfants avec la vie". L'église se trouvait à trois kilomètres, ce qui n'empêchait pas de s'y rendre, dimanches et fêtes, et pour la communion du premier vendredi du mois, y eût-il sur la route un mètre de neige.

Le pasteur de la paroisse, l'abbé Edmond Lessard, se faisait remarquer par sa piété et par sa science des voies spirituelles. $\mathrm{Au}$ catéchisme, il ne tarda pas à discerner l'enfant, qui unissait à l'amour des choses de Dieu une précoce intelligence. Zéphirin, de son côté, eut très tôt le désir de "ressembler à Monsieur le curé". Lors de la retraite de première communion, les prédications d'un frère mineur, le Père Anselme Fisher, modifièrent quelque peu la ligne d'horizon. L'idéal fut désormais de "faire comme saint Antoine", ce qui serait "encore plus beau". La maman acquiesça d'enthousiasme. Le père hésita davantage à se séparer de son aîné. Il finit par répondre au religieux qui plaidait la cause du petit et son envoi dans un séminaire franciscain: "Vous l'aurez, mais à une condition, c'est que vous le fassiez marcher droit."

Le 24 août 1902, l'enfant entre au collège tenu par les fils de saint François à Montréal. A cette époque, le Collège Séraphique était une simple résidence. Les aspirants franciscains suivaient leurs cours au Séminaire de Montréal, dirigé par les Sulpiciens. Il s'adapte d'emblée au régime, alors des plus sévères, et fait face aux études avec une aisance étonnante. Esprit profond, servi par une mémoire insolente, son nom s'inscrit en tête du palmarès. En même temps, il progresse en dévotion, sous l'influence du Père Célestin Demers, un fervent de 
l'ascète espagnol, saint Pierre d'Alcantara, et qui unit comme lui à l'extrémisme de la pénitence la bonté la plus communicative. A son école, il apprend à aimer le Patriarche de Nazareth, Patron du Canada; aussi, grande est sa joie quand la promenade hebdomadaire prend pour but l'Oratoire Saint-Joseph où l'on rencontre le frère André Bessette, "le Saint", comme on l'appelait déjà. À la même époque, un autre personnage de Légende Dorée, le Père Frédéric de Ghyvelde, dont la bure était partout populaire, faisait au Séminaire de rapides apparitions, dans un halo de prodiges et de vertus héroïques.

Rien ne semblait compromettre une telle vocation quand, à la fin de 1906 - c'était à l'âge de la croissance - l'organisme cède soudain. Épuisement nerveux, insomnie, vertiges, hémorragies obligent à renvoyer l'adolescent chez lui. Mais l'air salubre de la campagne ne lui rend pas la vitalité d'antan. Le chagrin le ronge; il s'étiole. Un nouvel essai d'études, en septembre 1907, doit être interrompu après trois mois. C'est le renvoi définitif. Mais Zéphirin ne s'y résigne pas. Il pleure, il prie, il s'accroche à son chapelet. La maman qui, silencieusement, partage sa peine, fait violence à la Vierge. Le jeune homme travaille à la ferme, mais son cœur est ailleurs.

En août 1908, une carte le convoque au Collège. Le Père Célestin-Joseph a son plan. Il ne peut croire à la faillite de si belles espérances. Il emmène son postulant obstiné au Sanctuaire de Saint-Joseph. Il le présente au Frère André Bessette. Mais laissons la parole au Père Ephrem en personne, puisqu'il a mis par écrit, à l'intention du biographe du "saint de Montréal", le témoignage oral qu'il m'avait donné sur sa guérison.

"Voici, dit le Père Célestin-Joseph en me désignant, l'un de mes grands élèves. Il a dû tout abandonner depuis deux ans. Il faudrait bien qu'il reprenne ses études... C'est pour cela que je l'ai amené près de saint Joseph." Le Frère André, me fixant tout droit et posant ses mains sur mes épaules familièrement, me dit: "Mais oui, mais oui, mon petit. Il faut reprendre. Tu seras prêtre. Tu ne seras plus malade pour t'arrêter encore." Puis il ajouta aussitôt: "Écoute bien ce que 
je vais te dire: tu vas le payer. Il faut payer les grâces du Bon Dieu. Cela, ne l'oublie pas !" Et après avoir proféré ces paroles ...: "Tiens, dit-il, prends cette médaille. Va prier à la statue de saint Joseph." Je me rendis de nouveau à la chapelle, et cette fois avec le Père Célestin. Nous priâmes quelques instants devant la statue de saint Joseph. Pendant ce temps-là, le Frère André était retourné à ce qui lui servait de bureau et de sacristie.

"Rentré très heureux au collège, j'étais tout entier à l'espérance. Mais ce soir-là, le Père Célestin ne me dit absolument rien en ce sens. Lu lendemain, après la messe que je lui servis dans la petite chapelle du Collège, il m'appela et me dit avec un sourire: "Tu vas rester au Collège et reprendre les études. Ce matin, j'écris à ton papa. Les paroles du Frère André se sont toutes réalisées."

C'est ainsi que fut rouverte "la marche des degrés" qui conduit à l'autel du Seigneur. Ses humanités achevées, le jeune homme prit l'habit franciscain, le 15 août 1911, sous le nom de Frère Ephrem. Le 25 août 1912, il fera ses vœux temporaires; le 28 août 1915, sa profession solennelle. Ses études de philosophie et de théologie, au scolasticat de Québec, révélèrent en lui un travailleur acharné et déjà chercheur qui entendait aller au fond des problèmes. Il ne craignait pas d'aborder Duns Scot dans le texte, sans se laisser décourager par la hardiesse du raisonnement, ni par les obscurités d'un style volontiers elliptique, qui requiert toute une initiation. Il pleurait parfois, impuissant à franchir l'obstacle, puis s'agenouillait et priait l'Esprit-Saint de l'éclairer. C'est à coups d'efforts douloureux qu'il acquit sa belle maîtrise de la pensée du Docteur subtil. Cela lui valait les boutades du frère infirmier, près de qui il cherchait le soulagement des maux de tête qui le tenaillaient parfois. "Laissez Scot de côté, et cela vaudra tous les remèdes", lui disait ce brave cœur, qui n'avait rien d'un intellectuel.

Le 14 juillet 1918, en la fête de saint Bonaventure, qui était un de ses protecteurs préférés, notre étudiant accéda à la prêtrise. Un de ses frères, l'abbé Anselme Longpré, devait, 
lui aussi, plus tard, recevoir le sacerdoce, cependant que deux de ses sœurs se consacraient à Dieu, l'une chez les Franciscaines Missionnaires de Marie, l'autre chez les religieuses de la Présentation de Marie. Au pays de Maria Chapdelaine, de telles rencontres en un même foyer ne sont pas rares.

Le nouvel ordonné avait l'étoffe d'un travailleur de l'esprit. A l'automne de 1918, il entra à l'Institut Pontifical Oriental, récemment fondé par Benoît XV. Le 25 juillet 1920, il enlevait brillamment le doctorat en théologie, en soutenant une thèse, aussi étayée de textes que solidement charpentée, sur la Procession du Saint-Esprit chez les auteurs franciscains du treizième siècle. Chemin faisant, s'était éveillée et précisée cette vocation de médiéviste qui lui vaudra tant de succès. Peu après, on l'intègre au Collège Saint-Bonaventure de Quaracchi près de Florence. Fondé en 1877 , ce centre franciscain voué à l'étude des auteurs du Moyen Áge avait été mis en veilleuse pendant la première guerre mondiale. Il allait prendre un nouveau départ, unissant pour un travail en équipe des spécialistes de différentes disciplines. C'est là que notre religieux mettra au point ses méthodes et s'imposera par ses compétences heureusement harmonisées d'historien, de théologien, de philosophe, et d'auteur spirituel.

Dès 1921 , lors de la célébration du septième centenaire du Docteur séraphique, une œuvre magistrale, La théologie mystique de Saint Bonaventure, attire sur lui l'attention des connaisseurs. Il est déjà l'érudit qui n'avance rien sans l'appuyer par des citations et des références, le bourreau de travail qui épuise la matière, l'écrivain aussi qui connaît son métier, sachant classer, composer, ordonner et parler une langue dont la clarté est la qualité principale, et qui ne manque ni d'éclat ni de relief.

Bientôt, l'occasion est offerte au Père Ephrem Longpré de mettre en œuvre ses dons de polémiste. Une thèse soutenue en Sorbonne, le 13 juin 1.922, par B. Landry, sur la philosophie de Duns Scot, malmenait le Docteur franciscain, avec un partipris évident de l'opposer en tout à saint Thomas, de l'apparenter à Guillaume d'Occam, et de le présenter comme "ami des spiri- 
tuels, ardent défenseur des droits de Dieu, mais qui a perdu le sens de l'amour". Pour qui connaissait la doctrine scotiste, l'erreur était de taille. Cette charge injuste excita "l'irritation du Père Ephrem". Le mot est d'Étienne Gilson, qui explique les contresens par la confusion des plans philosophique et théologique. Cela nous valut une série d'articles, réunis en volume, en 1924, sous le titre: La philosophie du Bienheureux Duns Scot. L'auteur, dans la conclusion, réclamait "que l'on inaugure enfin, lorsqu'on parle de l'École franciscaine, la méthode scientifique, objective, impartiale, en honneur partout dans les autres domaines". Cette réplique eut dans tous les milieux philosophiques et théologiques un grand retentissement. Elle valut au Père Ephrem des amitiés profondes et durables: celles, entre autres, du Cardinal Ehrle, de M. Maurice de Wulf, de Mgr Martin Grabmann et de M. Etienne Gilson lui-même, un des analystes les plus réputés de la pensée médiévale.

Le nom de notre religieux est désormais en vedette. Il s'attelle à l'édition critique de la Somme Théologique d'Alexandre de Halès, le maître de l'Aquinate et de saint Bonaventure. Sollicité de partout, il apporte sa contribution aux revues scientifiques franciscaines, aux centres intellectuels catholiques, aux grands Dictionnaires de théologie, d'histoire et de géographie ecclésiastique, de spiritualité ascétique et mystique. Un article sur Raymond Lulle attire l'attention des savants sur ce précurseur. Quand sera publiée, en 1934, une édition mineure de textes bonaventuriens, c'est encore au Père Ephrem qu'on recourra pour rédiger la préface générale. Elle n'est pas signée, mais l'inspiration ne trompe pas, ni la griffe, ni le coup de griffe.

Un chapitre capital de son activité est la part prise par lui à la préparation de l'édition critique des œuvres de Duns Scot. Dès novembre 1923, il est chargé de réunir à cette fin le matériel nécessaire. En juin 1927, il est nommé premier Préfet de la Commission responsable de cette publication. Entre 1928 et 1938, il visite systématiquement presque toutes les grandes bibliothèques de l'Europe. Il faut dire qu'il était de la race de ces Christophe Colomb d'archives, dont le flair et les intuitions 
excellent à découvrir des trésors dans l'amoncellement des documents où d'autres se découragent et s'égarent.

Il visite l'Angleterre et l'Écosse, où il découvre de très importants documents sur la carrière doctrinale de Duns Scot; l'Espagne et le Portugal, où des trésors insoupçonnés s'ouvrent à ses yeux émerveillés; la France et l'Italie si riches en trésors scientifiques qu'on ne saurait jamais se vanter de les avoir complètement inventoriés; la Suisse, la Belgique et l'Allemagne, enfin, accueillent tour à tour ce chercheur infatigable. Au printemps de 1938, peu de jours avant que la Commission scotiste ne fut transportée à Rome, le P. Victorin Doucet, assistant du $P$. Ephrem, pouvait dresser une liste de près de 450 manuscrits des œuvres de Duns Scot, dont la plupart avaient été repérés et décrits pour la première fois par le $\mathrm{P}$. Longpré lui-même.

En outre, les plus importants de ces manuscrits avaient été photographiés en tout ou en partie et formaient un ensemble de près de 35,850 photogyraphies, dont pas moins de 29,650 des œuvres mêmes de Scot. Il ne devait pas récolter le fruit de son labeur. La nouvelle Commission scotiste s'empara de cet immense butin. Le P. Ephrem Longpré fut éliminé. Il est hors de notre propos d'exposer ici les raisons secrètes de ce drame. L'historien de l'avenir en trouvera le récit détaillé dans les Mémoires du P. Longpré. Ce fut pour lui la grande épreuve, dont il porta jusqu'au bout la secrète blessure. En toute existence qui monte vers Dieu, il y a le glaive de la purification, et qui frappe au point sensible. Ce serviteur de la pensée fut atteint au cœur même de son œuvre.

Du même coup, il quittait le secteur apostolique auquel il avait consacré une part de ses énergies. Le penseur en lui ne supprimait pas le pasteur. Pendant ses temps libres, il exerçait volontiers son ministère dans les milieux les plus pauvres. Je garde la mémoire des traits qu'il me contait avec amour, vrais fioretti de l'âge moderne: cette adolescente qu'il avait instruite, baptisée, conduite à un ha.ut degré d'union à Dieu, vrai lis dans un bourbier, et qu'il communierait et aiderait à mourir, dans le bouge où elle vivait. C'es jeunes gens, venus en railleurs au 
passage du Saint-Sacrement, la casquette vissée sur la tête, et soudain terrassés par la grâce, jetés à genoux et décoiffés, qu'il guida jusqu'à l'autel de leur première messe. Ces souvenirs, à distance, lui arrachait des larmes.

En 1938, le Père Général l'autorise à se fixer à Paris, capitale intellectuelle, centre unique de documentation et d'échanges, où, sur des pistes nouvelles, il poursuivra ses travaux. Il sera désormais une des figures les plus stables et les plus familières du couvent de la rue Marie-Rose. Sans rien renier de son culte pour la patrie canadienne dont il était légitimement fier, il aimera passionnément son nouveau pays d'adoption. Il ne changera de résidence que sous l'occupation allemande, où, poursuivi par la Gestapo, il gagnera le Sud. Réfugié à Pau pendant deux ans, il prendra une part active à la Résistance, ce qui lui vaudra deux décorations de guerre, l'une française, l'autre anglaise. La Libération lui permettra de réintégrer sa cellule et de continuer sans encombre sa studieuse carrière.

Le moment est venu de camper le Père Ephrem Longpré, tel que je l'ai connu dans les dernières vingt-cinq années de son existence si remplie. De taille moyenne, avec une forte musculature et l'embonpoint que donne la vie sédentaire, il impressionnait, au premier regard, par la tête puissante, le front d'une magnifique ampleur, un crâne capable de contenir plusieurs cerveaux. La mâchoire était quelque peu proéminente et s'animait d'une moue volontaire quand il s'agissait de défendre des idées chères et des principes essentiels. Les lèvres, volontiers serrées, se détendaient parfois en un rire homérique. Il parlait rapidement, pas toujours distinctement, s'animant comme s'il livrait bataille à quelque interlocuteur invisible. Un masque de penseur et de lutteur, mais avec quelque chose de candide, voire de naif, comme d'un enfant.

Sa conversation était prodigieusement intéressante, truffée de citations, d'anecdotes, de dates, qu'une mémoire extraor- 
dinairement fidèle étiquetait, cataloguait et exhibait en temps opportun, avec une sûreté infaillible. Dans le bureau où il se carrait, comme en une citadelle, entouré de papiers et de livres montant à l'assaut de toutes parts, il semblait diriger la manœuvre, dégageant prestement tel classeur, compulsant tel dictionnaire, allant droit à telle brochure enfouie au sommet des rayons. On sentait qu'un ordre implacable présidait à la distribution des idées, des faits et des choses. Le Père Ephrem avait le culte du labeur. Il n'en avouait pas moins qu'à certaines heures de fatigue ou de lassitude morale, sa grande mortification était de se fixer sur ses dossiers, s'interdisant toute évasion.

Cela ne l'empêchait pas de se montrer d'une extrême complaisance quand vous alliez le déranger en pleine besogne. Que de fois ne m'a-t-il pas aidé à dénicher l'ouvrage approprié, à retrouver une citation, à mettre au point une pensée ! Aux lettres sollicitant des renseignements, il répondait aussitôt, longuement, avec précision, prodiguant sa science sans pose ni réticence. Il était conscient de l'immensité de son savoir, de la sécurité de ses méthodes, mais il en faisait hommage à Dieu en toute humilité, et il en parlait simplement, comme s'il se fût agi d'un autre. S'il lui arrivait parfois de garder trop longtemps des livres empruntés, il prêtait volontiers les livres et les documents à son usage et ne s'en montrait nullement jaloux. Combien de prêtres, de laîcs et de religieux de toutes communautés lui doivent une large part de leur succès dans la préparation de leurs doctorats. $\AA$ A preuve les quinze thèses manuscrites trouvées dans son bureau et la bonne centaine de bouquins dédicacés par les auteurs, en remerciement de ses services. Cet homme d'étude ne savait pas se refuser à qui sollicitait son assistance pour un travail intellectuel.

Hypersensible, très émotif, parfois jusqu'à la nervosité, souffrant vivement des propos et des gestes où il croyait voir une intention peu bienveillante, le Père Ephrem se montrait d'une reconnaissance expansive pour la moindre marque d'amitié. Il avait le cœur fidèle. Au fond, cet intellectuel était un grand affectif, et le contraste ne manquait pas d'étonner. C'est 
pourquoi, loin de le dessécher, sa science "se tournait à aimer", selon le mot de Bossuet. Il était en cela un vrai fils de saint François, et il considérait comme une consigne adressée à lui personnellement la lettre par laquelle le Poverello précisait à saint Antoine de Padoue le sens de sa mission doctrinale: "Il me plaît que tu enseignes aux frères la sainte théologie, à condition que ceux qui se livrent à cette étude n'éteignent pas en eux l'esprit de sainte oraison et de consécration à Dieu, comme il est marqué dans la Règle."

Cette Règle, le Père Ephrem la mettait en place d'honneur dans sa cellule, en ce coin de mystère où une lampe toujours allumée veillait l'image du Christ et celle de la Vierge. Là, il se recueillait à genoux, avec l'âme d'un tout petit, car il y avait en lui tout un côté d'enfance qui rayonnait dans sa piété. Son principal pôle d'attraction était l'Eucharistie: le saint Sacrifice d'abord, et aussi la station devant le Tabernacle, à laquelle il ne manquait jamais. A chaque férie libre, il célébrait la messe du Saint-Sacrement. Volontiers sentimentale en ses manifestations, sa dévotion était doctrinale en son inspiration fondamentale, nourrie du Docteur subtil et de saint Bonaventure, axée essentiellement sur la primauté de la charité et l'identification au Christ par l'intervention des dons du Saint-Esprit. La thèse scotiste, qui lui était si chère, de l'Incarnation voulue avant le péché et indépendamment de lui, pour la gloire du Père et l'effusion de sa tendresse, confère à la Vierge, dans le plan de la création, un rang et un rôle d'une beauté singulière. Cela donnait à la piété mariale de notre religieux un accent de profondeur qui, loin d'en altérer la confiance filiale, la poussait au plus haut point de l'abandon. Il eût voulu n'être dépassé par personne dans son amour pour la Madone. Chaque samedi où les rubriques l'autorisaient, il usait du privilège franciscain de dire la messe de l'Immaculée.

A Paris comme à Quaracchi, le Père Ephrem avait son rayon de ministère. Le labeur intellectuel ne le coupait pas de l'action; il l'alimentait en substance. Je puis témoigner qu'en lui le confesseur ne se bornait pas à une absolution prestement 
donnée après un bref fervorino passe-partout. S'inspirant des besoins personnels du pénitent comme des orientations liturgiques, c'est un vrai thème d'oraison qu'il suggérait. Toute son âme y passait. Sa dignité, son onction impressionnaient.

Dès 1939, il avait accepté d'assurer l'aumônerie de la grande maison des Franciscaines Missionnaires de Marie, avenue Reille. Il la gardera, sauf l'intermède de l'exil à Pau, jusqu'aux derniers mois de sa vie, où la maladie lui rendra ce service impossible. Chaque matin, par tous les temps et quelle que fût sa fatigue, il arrivait, à six heures, dans la chapelle, dont il aimait le recueillement. Il se préparait pour la messe de six heures trente. Le soir, il revenait pour le salut de dix-huit heures. Sa fidélité, son exactitude étaient sans faille, comme son attachement à l'Institut, qu'il aimait pour son caractère dynamique, et aussi et surtout, parce qu'il pratique l'Adoration perpétuelle du SaintSacrement.

J'ai sous les yeux ure série de témoignages recueillis près des religieuses qui l'ont le mieux connu. Il y règne une unanimité touchante pour louer son exquise bonté, son dévouement hors de pair. Il était toujours disponible pour confesser, pour porter la communion aux infirmes. On apprécie le caractère apaisant de sa direction, les conseils qu'il donnait à telle ou telle Sœur pour faciliter ses études, sa façon miséricordieuse d'encourager, qui sauva plus d'une vocation. Volontiers, il faisait appel aux motifs d'apostolat. Dans ses conférences, l'Eucharistie et la Passion, l'Agonie de Jésus surtout, constituaient, avec la Sainte Vierge, les thèmes préférés. A plusieurs reprises, on le pria de rendre visite à quelque mourante qui refusait les sacrements. La diplomatie de la charité lui ouvrait toutes les portes. Il considérait comme une grâce d'aider les âmes proches du trépas.

Sa piété expansive parlait par elle-même. Jamais il ne passait devant la statue de Marie sans s'incliner et se recueillir. Il aimait prolonger son adoration devant le Saint-Sacrement. C'est ce qui l'attirait che:z les Franciscaines, les jours mêmes où quelque hôte de passage leur assurait la messe. Lors de la 
communion portée aux malades, il ne les quittait jamais sans approcher d'elles le ciboire pour les bénir. Au Domine non sum dignus, et quand il s'inclinait sur l'Hostie, il semblait comme absorbé et subjugué par la Divine Présence. Ce ministère le fatiguait parfois. Il lui arrivait de dire qu'il était temps de songer à le remplacer. Mais chacun savait qu'il y tenait comme à la prunelle de ses yeux et qu'il n'y renoncerait que pour mourir, ce qui se réalisa effectivement. Les Franciscaines Missionnaires, en l'entourant dans sa dernière maladie, lui paieront un noble tribut de gratitude.

Parmi les multiples ouvrages dus à la plume du Père Ephrem, celui qu'il porta toute sa vie dans la pensée et qui peut-être lui tint le plus à cœur, car alliant la piété au savoir, ce fut l'article publié dans le Dictionnaire de Spiritualité, sous le titre: Eucharistie et Expérience Mystique. Il ne sortit qu'en 1962, mais trente ans de recherches l'avaient préparé. La thèse revient à ceci: “Deux voies conduisent à l'expérience mystique. La première, d'ordre surtout psychologique, est la contemplation. Cet itinéraire a été décrit par les plus hauts mystiques: Bonaventure, Jean de la Croix, Thérèse d'Avila... La seconde voie, la voie sacramentelle et liturgique, a été, au contraire, presque totalement négligée, en dépit des essais de systématisation tentés par Thomas de Jésus et ses disciples, et ce fut une surprise, dans la plupart de milieux spirituels modernes, lorsque, sur les indications de saint Bonaventure, le sentiment qui voit dans l'Eucharistie la seconde source de l'expérience mystique fut proposé explicitement." En invoquant l'Écriture et la tradition, en citant longuement théologiens et contemplatifs - ce qui suppose une immense lecture - l'auteur établit que "l'Eucharistie est le principe sacramentel de l'expérience mystique, de ses débuts à ses plus hauts sommets". Il conclut d'ailleurs que "oraison active ou passive et communion se soutiennent mutuellement; elles s'associent dans un même itinéraire vers la Trinité par le Christ Jésus ..." La principale difficulté du travail avait été de condenser en trente-trois colonnes une 
matière presque illimitée. C'est peut-être l'œuvre écrite dont notre franciscain se satisfaisait le plus. Il y avait livré le meilleur de lui-même.

Dans le même Dictionnaire de Spiritualité avait paru, signée du Père Ephrem, une étude sur la Contemplation dans l'école franciscaine. Un article plus volumineux encore - il comportait soixante-quinze colonnes - avait été consacré par lui à saint Bonaventure. Après un bref aperçu biographique et un relevé sommaire des écrits spirituels de son héros, l'auteur analysait longuement sa doctrine mystique, s'attardant notamment à la théorie des trois voies et donnant toute sa mesure dans l'exposé de la vie contemplative, de ses formes diverses et des dispositions qu'elle requiert. Il achevait sur un ton presque triomphal où vibrait toute sa foi: "La doctrine de saint Bonaventure est tout entière un appel à la contemplation et à l'action. Dans l'histoire religieuse des temps, il ne s'est jamais peut-être trouvé un Saint qui ait fait entendre au monde une invitation aussi large et aussi pressante à l'union mystique que celle du séraphique Docteur. Pour ces raisons, entre beaucoup d'autres, la doctrine spirituelle de saint Bonaventure, tout imprégnée d'onction et de poésie, constitue un monument unique dans la littérature mystique. A l'époque où saint Bonaventure écrivait, l'élan vers les hauteurs et l'infini inspirait aux architectes gothiques le chœur sublime de la cathédrale de Beauvais. La doctrine mystique du séraphique Docteur est jaillie de la même inspiration. Elle est la montée vertigineuse vers Dieu, l'extase, la "sursumactio" la plus haute et la plus flamboyante de la pensée médiévale".

A côté des travaux de fond, il y avait ce que le Père appelait "les bluettes", articles de revues, production secondaire, collaboration mineure à de multiples Dictionnaires. Dans ses recherches aux Archives Nationales, il rencontrait, au passage, des documents de toutes sortes, et comme tout l'intéressait, il constituait des dossiers en vue d'une publication éventuelle. Il y avait quelque chose d'hallucinant à l'entendre énumérer ses projets, comme si l'éternité lui était acquise. Parmi ces sujets 
"possibles", et qui jamais ne verront le jour, se trouvait une étude sur les rapports qu'eut avec Jeanne d'Arc un franciscain, le Frère Richard. J'entends encore le Père Ephrem m'exposer, pendant plus d'une heure, avec force citations à l'appui, et ne recourant qu'à sa mémoire, une thèse d'allure révolutionnaire sur les cours qu'aurait suivis à l'Université de Paris saint Antoine de Padoue. Il y mettait une conviction qui n'avait d'égale que la rigueur de l'argumentation. Quelle passion aussi dans sa voix le jour où il me livra sa pensée sur les perspectives mystiques de la doctrine de Duns Scot et la profondeur de sa filiation franciscaine. Car il aimait passionnément sa famille religieuse, "l'Ordre", comme il disait, sans spécifier.

En 1955, c'est du Canada qu'on sollicite, une fois de plus, son concours. Le Père Ephrem n'eût pas été un vrai Canadien s'il n'avait voué au patriarche de Nazareth une tendresse de prédilection. Lors du Congrès, qui se tint, cette année-là, à Montréal, du 31 juillet au 9 août, et qui avait pour thème central le Patronage de saint Joseph, notre franciscain eut à étudier ce sujet "d'après l'école franciscaine du 13e siècle". Il fit aussi, à cette occasion, des recherches dans les archives du Vatican pour tenter de retrouver le décret d'Urbain VII qui avait contribué, au XVII ${ }^{e}$ siècle, à donner plus d'éclat à la fête du glorieux protecteur du Canada.

Jamais le Père Ephrem ne tolérait l'à-peu-près. Quand il envisageait un débat, quand il faisait un exposé ou écrivait ne serait-ce qu'un modeste article, il lui fallait tout voir et tout lire en la matière. Cela entraînait des délais, des retards de livraison, qui provoquaient parfois des conflits avec les responsables de la publication des Dictionnaires auxquels il collaborait. Ceux-ci - ce fut le cas notamment pour le directeur de Catholicisme - voyant leurs fascicules en panne venaient relancer notre écrivain trop consciencieux. Des brouilles surgissaient, suivies de réconciliations, quand il fallait, bon gré mal gré, mobiliser à nouveau sa compétence et qu'il s'acquittait de la tâche, avec sa maestria coutumière, à la satisfaction de tous les connaisseurs. Car, il faut le dire, dans le monde des 
médiévistes, historiens et théologiens, le Père Ephrem avait la cote. Sa signature faissiit autorité.

Si l'on songe qu'il a accompli, seul, une besogne qui en eût écrasé plusieurs, qu'il menait de front de nombreuses tâches, qu'il en projetait et en méditait plus encore, et cela malgré une santé de plus en plus délabrée, de misères physiques multiples: migraines, eczéma, hernie, faiblesse cardiaque, enflure des jambes, on ne peut que s'incliner devant cette puissance de travail et cette volonté d'acier. Il y a là un bel exemple pour les jeunes générations.

La Providence permit que l'ultime effort du savant fût appliqué à faire revivre saint François. Il s'agissait, cette fois encore, d'un article important pour le Dictionnaire de Spiritualité. Un certain nombre de religieux sollicités s'étant récusés, le Père Ephrem, qui s'était longtemps dérobé, en raison de sa fatigue croissante, finit par accepter la proposition. La curie généralice des Frères mineurs l'y encourageait fortement. Comme toujours, il prit son temps, incapable de fournir une copie facile où le sujet serait quelque peu escamoté. Il étudia longuement les sources, explora le cadre historique, compulsa tous les ouvrages où la figure du Poverello, son esprit et son œuvre étaient mis en relief.

Ce travail de défrichement, sous l'aiguillon de la maison Beauchesne, désireuse de sortir à temps son livret, aboutit à une manière de petit chef-d'œuvre. Délaissant les aspects secondaires, le Père Ephrem montre en François le disciple, l'imitateur et le héraut du Christ. Il trace un portrait, qui s'inspire essentiellement de la Legenda Major de saint Bonaventure, mais qu'il étoffe de notions biographiques, de remarques psychologiques et de considérations empruntées à la spiritualité comparée. Après un aperçu sur la bibliographie générale, l'état des sources du franciscanisme et du milieu qui l'a vu naître et se développer, l'auteur trace les différentes étapes de l'ascension franciscaine: rencontre du Christ, adhérence au Christ, vertus qui s'épanouissent à l'école du Christ. La partie la plus originale du texte a trait à la vie intérieure et mystique du Stigmatisé de 
l'Alverne. A ma connaissance, et sauf erreur, nulle autre étude n'a approfondi avec une telle acuité d'analyse et une telle maîtrise de pensée la question des rapports de François d'Assise et de Jésus.

Il fallut tailler largement dans les bonnes feuilles pour les ramener aux dimensions des trente-six colonnes allouées. Toutefois, il était prévu qu'un livre les reprendrait intégralement plus tard, en y joignant tous les développements que le Père avait dû s'interdire. L'ouvrage est déjà annoncé, chez Beauchesne, pour l'année 1966. La maladie lui arrache la plume des mains. Elle l'empêcha également d'écrire un exposé synthétique de la spiritualité franciscaine aux seizième et dix-septième siècles, pour lequel il avait découvert et analysé plus de deux cents volumes, la plupart ensevelis dans l'oubli. En dépit de la déception de la tâche inachevée, il estimera comme une des plus grandes grâces de sa vie d'avoir pu ainsi puiser aux trésors du franciscanisme et d'avoir vécu toute une période à contempler et évoquer celui que Lacordaire appelait "le sublime amoureux de Jésus-Christ". J'ai vu de près son allégresse quand il lui advenait de découvrir quelques fioretti restés jusqu'ici dans l'ombre et qui faisaient apparaître des nuances nouvelles du visage paternel. Il faut voir là une ultime préparation que Dieu lui ménageait avant qu'il ne rencontrât "notre Sœur la Mort".

Depuis plusieurs années, l'état de santé du Père laissait à désirer. Une terrible poussée d'eczéma l'avait paralysé pour un temps. "Je me supporte avec patience, disait-il; c'est tout ce que je puis faire." Vinrent ensuite des crises cardiaques, apparemment bénignes, mais qui épuisaient ses forces. En décembre 1963, il dut s'aliter et suspendre toute activité pour trois bons mois. C'était la première crise du mal qui devait l'emporter. S'il reprit ses tâches apostoliques et le labeur intellectuel, ce fut au ralenti, au prix d'efforts courageux et de sa volonté de mourir debout. À la mi-mai 1965, le P. Ephrem entrait à la clinique de la Croix-Saint-Simon, dirigée par les Franciscaines Missionnaires de Marie... 
Peu de temps après, un nouvel accès du mal provoquait de nouveau son hospitalisation...

C'est dans la nuit du 9 au 10 octobre que le Père Ephrem Longpré se rendit compte qu'il n'y avait plus d'espoir de guérison.

Les funérailles eurent lieu dans la chapelle de la rue MarieRose, le jeudi, 21 octobre à 8 h. 30, sans éclat, sans grande affluence, dans un recueillement et une ferveur qui eussent enchanté celui qu'on pleurait. Frères Mineurs et Franciscaines Missionnaires de Marie lui faisaient une escorte de fraternelle reconnaissance. Une concélébration se déroula, au rythme apaisant des mélodies grégoriennes.

STÉPHANE-JOSEPH PIAT, o.f.m. 